\title{
Journal of Neurorehabilitation
}

\section{Basic Neuroscience Research Inspires Neurorehabilitation and Vice Versa Halina Baran* and Berthold Kepplinger}

Karl Landsteiner Research Institute for Neurochemistry, Neuropharmacology, Neurorehabilitation and Pain Treatment Mauer, Austria

Starting in 1980 with basic neuroscience and neuropharmacology research on the kainic acid model for human temporal lobe epilepsy in Oleh Hornykiewicz's Lab in Vienna and later with research work on Tryptophan metabolism in Robert Schwarcz's Lab in Baltimore and now in my Lab at the Karl Landsteiner Research Institute Mauer I experienced and still enjoy the fruitful connection with clinical neuroscientists. Particularly the cooperation with Berth Kepplinger the past Chief of Neurological Departments of Hospitals in Mauer and Amstetten, Austrian provided a convincing strategy for the integration of both, basic neuroscience and neurorehabilitation research.

In 1880 Jean-Martin Charcot introduced the first therapeutic indication of exercise by using a shaking chair in Parkinson patients which lowered tremor and improved stability [1]. A marked deficit of dopaminergic neurotransmission and its significance with Parkinson's symptoms was discovered by Oleh Hornykiewicz in 1963 [2] and his suggestion to use L-DOPA for therapy became later a standard for Parkinson treatment [3].

Interestingly, 100 years after Charcot's suggestion it has been shown in experimental animal studies that dopamine synthesis and metabolism in the striatum is increased significantly in rats exposed to exercise [4]. Scientists have suggested that physical exercise might contribute to adjust extracellular dopamine levels within an adequate range. Running activities applied to animal model of Parkinson's disease showed also improvement of motor symptoms and caused less degeneration of the dopaminergic system [5].

Stochastic resonance therapy (SRT), a whole body vibration, is a novel method for rehabilitation of patients with various neurological and psychiatric disorders e.g. Parkinson disease [6,7], Alzheimer's [8], Multiple Sclerosis [9], post stroke spasticity [10], post traumatic brain damage [11], depression and schizophrenia [12,13], and also with low back pain [14]. The effectiveness of SRT in the treatment of these diseases or conditions is significant however the mechanism is still widely unknown.

Kepplinger et al. demonstrated that treadmill running significantly lowered serum kynurenic acid in rats [15]. A recent study on influence of SRT on tryptophan metabolism in the serum of healthy human subjects revealed lowered L-tryptophan, L-kynurenine and kynurenic acid levels, as well [16].

With respect to dopaminergic neurotransmission scientists described an enhancement of dopamine levels in the plasma after exercise in rat and healthy human subjects [17]. Revealed data are in correlation with the observation that endogenous kynurenic acid can control the extracellular levels of dopamine at least in rat striatum [18] and the interaction between increased kynurenic acid and lowered dopamine levels in the striatum has been indicated [18].

Interestingly, comparison between L-DOPA treatment and the physical exercise in patients with spinal injury revealed similar positive effect in both types of treatment [11] supporting an enhanced dopamine function during exercise. Consistent with this data, application of exercise to Parkinson's patients treated with L-DOPA increased significantly therapeutic efficiency [19]. The improvement of Parkinson symptoms due to SRT is transient, nevertheless the positive effect would confirm an advantage of SRT and suggest its therapeutic significance in the rehabilitation process. The combination of SRT with L-DOPA medication might have an impact on reducing L-DOPA medication and preventing dyskinesia, as observed by Parkinson's patients after 5 to 6 years of L-DOPA therapy.

Kynurenine aminotransferases which are responsible for kynurenic acid synthesis are widely distributed in the mammalian body. Lowering of kynurenic acid levels after SRT could be due to an activation of glia depressing factor (GDF), which has the ability to block kynurenine aminotransferases activities, as we described recently [20]. Since kynurenic acid is increased and GDF is reduced in CSF of Multiple Sclerosis patients, we speculate that application of SRT ameliorates clinical symptoms of Multiple Sclerosis patients due to GDF activation.

But, there are also data demonstrating discrepancies in respect to neurochemical changes after exercise, and these discrepancies are probably due to different kinds of exercise (running vs. sinusoidal vibration vs. vibration with stochastic resonance), different kind of parameters (amplitude, frequency) and duration of exercise and this gives a reason to further study experimentally and clinically for better output of exercise methods.

Repetitive transcranial magnetic stimulation (rTMS), a noninvasive brain stimulation technique has been suggested to be effective for treatment of depression [21] and might reduce spasticity. In the last decade rTMS gained more attention and significance for neurorehabilitation. Our study on the influence of rTMS on patients with stroke, with central neuropathic pain and with major depression revealed clinical improvements, data presented in part [22]. Parallel to clinical investigation we performed measurement of tryptophan metabolites in the serum to find correlations between clinical outcome and neurochemical findings. Furthermore, our data suggest that the localisation and pattern of stimulus as well as the time period between rTMS and occupational therapy seems to be important for the clinical outcome, at least for stroke patients.

Increased kynurenic acid formation in the brain has been found in patients with neurodegenerative and neuroinflammatory disorders and certain psychiatric conditions, such as Alzheimer's, Parkinson's [23], HIV-1 encephalopathy [24] and Schizophrenia [25] or during the aging process [26]. These and also other accumulated data strongly suggest that increased kynurenic acid in the brain plays a role in the impairment of cognition and memory.

Kynurenic acid is a well-known endogenous antagonist of the

*Corresponding author: Halina Baran, Ph.D, Professor of Neurochemistry, Karl Landsteiner Research Institute for Neurochemistry, Neuropharmacology Neurorehabilitation and Pain Treatment Mauer, Austria, Tel: 00437475501 3600, 00436644436 169; Email: halina.baran@neuro-lab.eu

Received January 20, 2014; Accepted January 22, 2014; Published January 24, 2014

Citation: Baran H, Kepplinger B (2014) Basic Neuroscience Research Inspires Neurorehabilitation and Vice Versa. Int J Neurorehabilitation 1: e102. doi:10.4172/2376-0281.1000e102

Copyright: (c) 2014 Baran H. This is an open-access article distributed under the terms of the Creative Commons Attribution License, which permits unrestricted use, distribution, and reproduction in any medium, provided the original author and source are credited. 
glutamate ionotropic excitatory amino acid receptors N-methyl-Daspartate (NMDA), alpha-amino-3-hydroxy-5-methylisoxazole-4propionic acid (AMPA) and kainate [27] and of the nicotine cholinergic subtype $7 \alpha$ receptors [28] and exerts anticonvulsive and neuroprotective activities, at least in animal models of neurodegenerative diseases [27]. Therefore it is reasonable to believe that pharmacological approaches which influence kynurenic acid synthesis can affect neurotransmission in the brain and have an impact on cognition and memory.

Indeed, Cerebrolysin, a porcine brain extract with neurotropic activities is used for the treatment of Alzheimer's to improve brain activities, memory and cognition, and it blocks cerebral kynurenic acid synthesis, at least in an in vitro study [29]. Interestingly, D-Cycloserine, an anti-mycobacterial drug, also blocks markedly kynurenic acid synthesis, what we discovered recently [30]. Importantly, this drug shows improvement of dementia in Alzheimer's patients and amelioration of cognition and/or delusion in schizophrenia [31].

Summarizing, new physical and pharmacological trials for neurorehabilitation are challenges for basic scientists to help clearing the mechanism(s) of action and to find possible clues for more successful applications. On the other hand the knowledge of neurochemical and neuropharmacological findings are fruitful for professionals of rehabilitation to provide more understandings of rehabilitative interventions, which may guide to reasonable selection of treatment options.

\section{References}

1. Goetz CG (2009) Jean-Martin Charcot and his vibratory chair for Parkinson disease. Neurology 73: 475-478.

2. Hornykiewicz $O$ (1963) The tropical localization and content of noradrenalin and dopamine (3-hydroxytyramine) in the substantia nigra of normal persons and patients with Parkinson's disease. Wien Klin Wochenschr 75: 309-312.

3. Hornykiewicz O (1994) Levo-dopa in the 1960s: Starting point Vienna. In: Poewe W, Lees AJ, editors. 20 Years of Madopar - New Avenues. Basle, Switzerland: Editiones Roche; 1994

4. Nakamura H, Moroji T, Nohara S, Nakamura H, Okada A (1992) Activation of cerebral dopaminergic systems by noise and whole-body vibration. Environ Res 57: 10-18.

5. Tillerson JL, Cohen AD, Philhower J, Miller GW, Zigmond MJ, et al. (2001) Forced limb-use effects on the behavioral and neurochemical effects of 6-hydroxydopamine. J Neurosci 21: 4427-4435.

6. Haas CT, Turbanski S, Kessler K, Schmidtbleicher D (2006) The effects of random whole-body-vibration on motor symptoms in Parkinson's disease. NeuroRehabilitation 21: 29-36.

7. Hirsch MA, Farley BG (2009) Exercise and neuroplasticity in persons living with Parkinson's disease. Eur J Phys Rehabil Med 45: 215-229.

8. Pitkala KH, Raivio MM, Laakkonen ML, Tilvis RS, Kautiainen H, et al. (2010) Exercise rehabilitation on home-dwelling patients with Alzheimer's disease-a randomized, controlled trial. Study protocol. Trials 6: 11: 92.

9. Wunderer K, Schabrun SM, Chipchase LS (2010) Effects of whole body vibration on strength and functional mobility in multiple sclerosis. Physiother Theory Pract 26: 374-384.

10. Kluding PM, Tseng BY, Billinger SA (2011) Exercise and executive function in individuals with chronic stroke: a pilot study. J Neurol Phys Ther 35: 11-17.

11. De Mello MT, Esreves AM, Tufik S (2004) Comparison between dopaminergic agents and physical exercise as treatment for periodic movements in patients with spinal cord injury. Spinal Cord 42: 218-221.
12. Lavie CJ, Milani RV, O'Keefe JH, Lavie TJ (2011) Impact of exercise training on psychological risk factors. Prog Cardiovasc Dis 53: 464-470.

13. Kucyi A, Alsuwaidan MT, Liauw SS, McIntyre RS (2010) Aerobic physical exercise as a possible treatment for neurocognitive dysfunction in bipolar disorder. Postgrad Med 122: 107-116.

14. Waller B, Lambeck J, Daly D (2009) Therapeutic aquatic exercise in the treatment of low back pain: a systematic review. Clin Rehabil 23: 3-14.

15. Kepplinger B, Kalina P, Zeiner D, Eigner S, Baran H (2007) Influence of exercise on kynurenic acid levels in the serum. Amino Acids 33: LVII.

16. Kepplinger B, Baran H, Sedlnitzky-Semler B, Badawi NR, Erhart H (2011) Stochastic resonance activity influences serum tryptophan metabolism in healthy human subjects. Int J Tryptophan Res 4: 49-60.

17. Yoshizumi M, Nakaya Y, Hibino T, Nomura M, Minakuchi K, et al. (1992) Changes in plasma free and sulfoconjugated catecholamines before and after acute physical exercise: experimental and clinical studies. Life Sci 51: 227-234.

18. Wu HQ, Rassoulpour A, Schwarcz R (2007) Kynurenic acid leads, dopamine follows: a new case of volume transmission in the brain? J Neural Transm 114: 33-41.

19. Muhlack S, Welnic J, Woitalla D, Muller T (2007) Exercise improves efficacy of levodopa in patients with Parkinson's disease. Mov Disord 22: 427-430.

20. Baran H, Kepplinger B, Draxler M (2010) Endogenous kynurenine aminotransferases inhibitor is proposed to act as "Glia Depressing Factor" (GDF). Int J Tryptophan Res 3: 13-22.

21. Schutter DJ, van Honk J (2005) A framework for targeting alternative brain regions with repetitive transcranial magnetic stimulation in the treatment of depression. J Psychiatry Neurosci 30: 91-97.

22. Kepplinger B, Sedlnitzky-Semler B, Eigner S, Kalina P, Baran H (2013) Quality management of magnetic resonance imaging (MRI) guided repetitive transcranial magnetic stimulation (rTMS) for the treatment of major depression. Soc Neurosci Abstr 503.07.

23. Baran H, Jellinger K, Deecke L (1999) Kynurenine metabolism in Alzheimer's disease. J Neural Transm 106: 165-181.

24. Baran H, Hainfellner JA, Kepplinger B, Mazal PR, Schmid H, et al. (2000) Kynurenic acid metabolism in the brain of HIV-1 infected patients. J Neural Transm 107: 1127-1138.

25. Erhardt S, Blennow K, Nordin C, Skogh E, Lindstrom LH, et al. (2001) Kynurenic acid levels are elevated in the cerebrospinal fluid of patients with schizophrenia. Neurosci Letters 313: 96-98.

26. Kepplinger B, Baran H, Kainz A, Ferraz-Leite H, Newcombe J, et al. (2005) Age-related increase of kynurenic acid in human cerebrospinal fluid: Positive correlation with IgG and B2-microglobulin changes. Neurosignals 14(3): 126135.

27. Stone TW (1993) Neuropharmacology of quinolinic and kynurenic acids Pharmacol Rev 45: 309-379.

28. Hilmas C, Pereira EFR, Alkondon M, Rassoulpour A, Schwarcz R, et al. (2001) The brain metabolite kynurenic acid inhibits $\alpha 7$ nicotinicreceptor activity and increases non- $\alpha 7$ nicotinic receptor expression: Physiopathological implications. J Neurosci 21: 7463-7473.

29. Baran H, Kepplinger B (2006) Cerebrolysin lowers kynurenic acid formation- an in vitro study. Eur Neuropsychopharmacol 19: 161-168.

30. Baran H, Kepplinger B (2013) D-cycloserine lowers kynurenic acid formationNew mechanism of action. Eur Neuropsychopharmacology [doi: 10.1016/j. euroneuro.2013.10.006].

31. Goff DC (2012) D-cycloserine: an evolving role in learning and neuroplasticity in schizophrenia. Schizophr Bull 38(5): 936-941. 